\title{
Peripheral Ulcerative Keratitis: Another Ocular Manifestation of Behçet's Disease
}

\author{
Moctar Issiaka $^{1 *}$, Soukaina Bahti ${ }^{1}$, Adil Mchachi ${ }^{1}$, Leila Benhmidoune ${ }^{1}$, Rayad Rachid ${ }^{1}$, Mohamed El \\ Belhadji ${ }^{1}$ \\ ${ }^{1}$ Adult Ophthalmology Department, $20^{\text {th }}$ August Hospital, CHU Ibn Rochd, BP 2698, Casablanca, Morocco \\ *Corresponding Author: Moctar Issiaka, Adult Ophthalmology Department, $20^{\text {th }}$ August Hospital, CHU Ibn \\ Rochd, BP 2698, Casablanca, Morocco; Email: issiakamoctar@gmail.com
}

Received Date: 24-11-2021; Accepted Date: 17-12-2021; Published Date: 24-12-2021

Copyright $^{\bigcirc} 2021$ by Issiaka M, et al. All rights reserved. This is an open access article distributed under the terms of the Creative Commons Attribution License, which permits unrestricted use, distribution and reproduction in any medium, provided the original author and source are credited.

\begin{abstract}
Ocular involvement in Behcet's Disease (BD) is typically characterized by uveitis, which is present in over $50 \%$ of cases. However, more and more cases of corneal involvement are reported. We report a rare case of Peripheral Ulcerative Keratitis (PUK) associated with Behçet's disease. This is a 38-year-old man who has been followed for Behçet's disease for 5 years, in remission and without treatment for one year and without overt anterior ocular involvement, who presented a deep ulcerative lesion with a peri-lesional disorder in the nasal peripheral cornea of the left eye on a background of moderate dry eye. Initial visual acuity was $13 / 20$ without correction and there was no anterior chamber reaction or posterior segment involvement. Because of the worsening of the ulcer despite 3 days of treatment with antibiotics and antivirals, the diagnosis was reconsidered given the patient's history of BD and a course of topical and oral corticosteroids and wetting agents were instituted. The evolution was spectacular, after 5 days of treatment, with visual acuity back to 20/20 and almost complete healing, thus confirming the etiology of this keratitis. Any marginal ulcer, especially one that is refractory to antibiotics and antivirals, should be investigated for Behçet's disease.
\end{abstract}

\section{Keywords}

Keratitis; Dry Eye; Behçet's Disease; Matrix Metalloproteinase; Corticosteroid 


\section{Introduction}

Behçet's Disease (BD) is a multisystem disease characterized by skin lesions, recurrent oral aphthous ulcers, genital ulcers and ocular lesions [1]. The ocular manifestation of BD is becoming increasingly diverse, although uveitis is still the most common ocular manifestation, accounting for $50-85 \%$ of the total and remains a leading cause of morbidity. Pure corneal involvement is rare and presents as Peripheral Ulcerative Keratitis (PUK) [2]. The latter results from unilateral stromal inflammation in the form of a marginal ulcer and is the initial manifestation of collagen vascular diseases in $50 \%$ of cases [3].

The aim of this work is to report an atypical ophthalmologic manifestation of BD: marginal corneal ulcer.

\section{Case Report}

This is a 38-year-old patient who has been followed for 5 years for Behçet's Disease, is currently in remission without any treatment for one year, who presented to the emergency consultation for a painful red left eye associated with photophobia. The diagnosis of BD was made in view of a history of previously repeated uveitis, recurrent oral and genital aphthosis and erythema nodosum on the legs and positive HLA B51 typing. On ophthalmological examination of the left eye, the visual acuity without correction was 13/20. The ocular tone was measured at $17 \mathrm{mmHg}$ with a forced-air tonometer. In the anterior segment, there is the presence of a peri-keratotic circle, a marginal ulcer in the inferior nasal area measuring $3 \times 2$ $\mathrm{mm}$, distant $2 \mathrm{~mm}$ from the limbus, stromal infiltration and a diffuse superficial punctate keratitis. This ulcer is deep in the center, clean, well delimited with slightly edematous margins and totally impregnated with fluorescein (Fig. 1). The tear break-up time was altered with a tear film break-up at $4 \mathrm{sec}$ and the Shirmer test was positive. There was no anterior chamber reaction, no evidence of uveitis with a clear lens and an unremarkable fundus. The contralateral eye examination was unremarkable, with an uncorrected visual acuity of 20/20 and mild dry eye. The patient underwent a corneal scraping for biological study and was put on local broadspectrum antibiotics (Fluoroquinolone) and wetting agents. The evolution was marked at 48 hours by the extension of the ulcer and the corneal sample was sterile. Given this context and the patient's history, the immunological mechanism was evoked and the patient was put on local corticosteroids (hourly instillation for 48 hours, then 6 times a day) and oral corticosteroids (20 mg/kg/day), as well as wetting agents with close monitoring. The improvement was then spectacular after 24 hours, with complete healing of the ulcer in less than 5 days (Fig. 2), with a visual acuity of 20/20 without correction. The corneal damage was considered a reactivation of his Behçet's disease and he was readmitted to his internist to adapt to his treatment. 

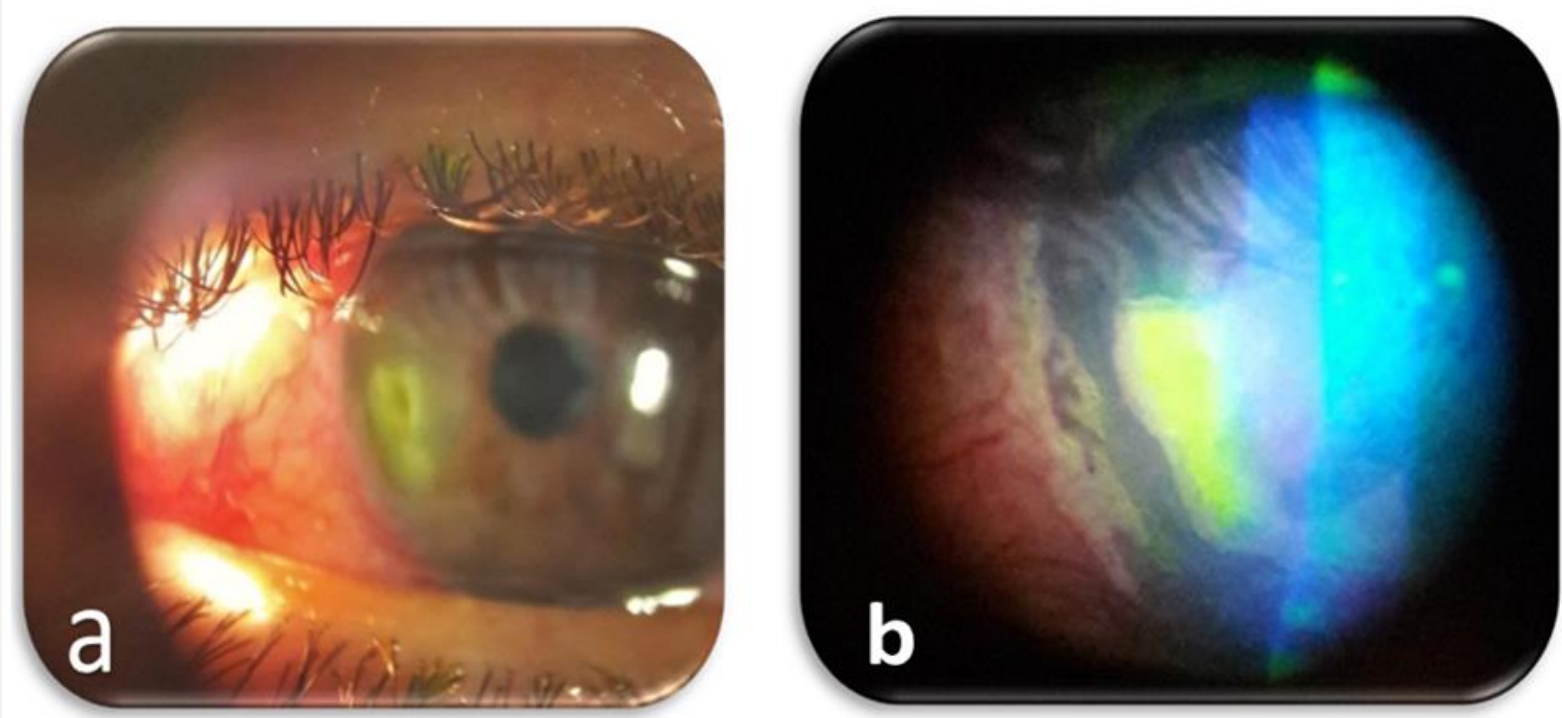

Figure 1: Nasal marginal ulcer (a) with diffuse superficial punctate keratitis and fluorescein intake (b).
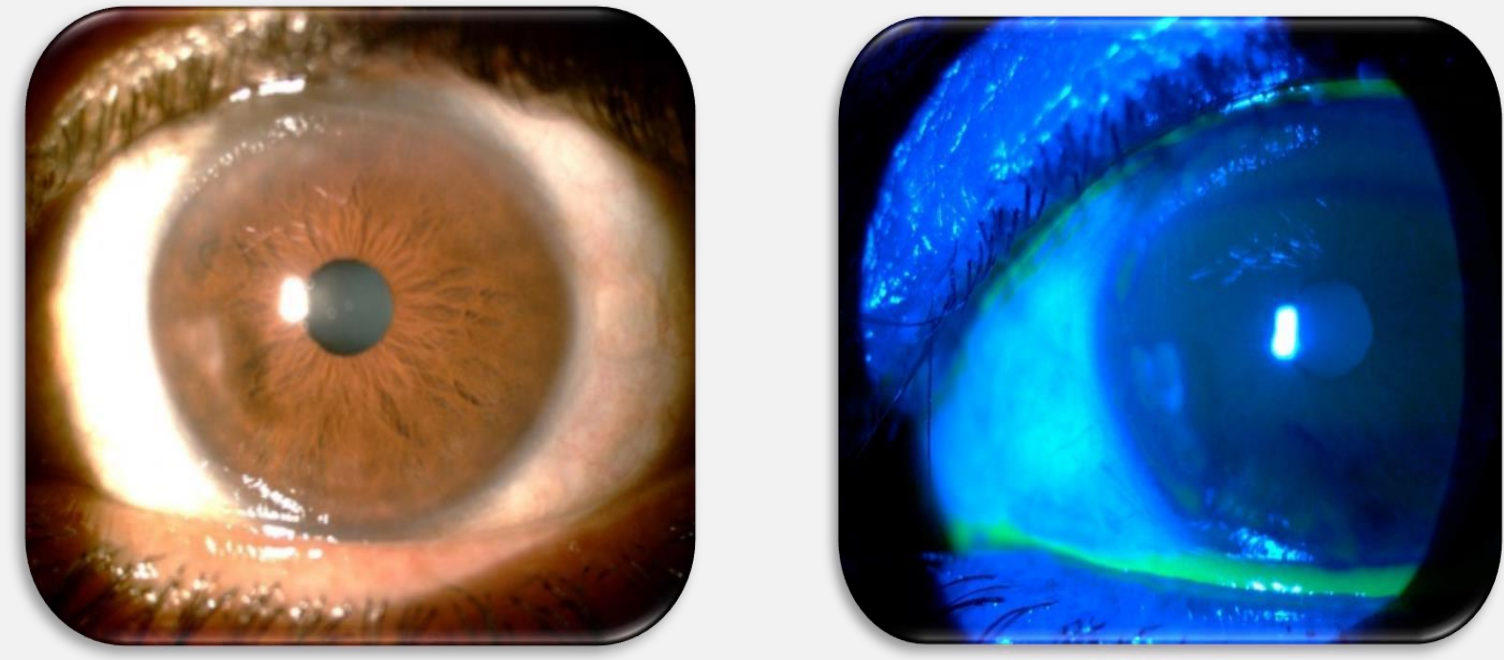

Figure 2: Appearance after 5 days of corticosteroid therapy, good healing with improved tear quality.

\section{Discussion}

Behcet's Syndrome (BS) is a vasculitis that causes oral and vaginal ulcers, skin lesions, uveitis and vascular, central nervous system and gastrointestinal involvement. It is more frequent in the Mediterranean and the Far East [4]. Ocular involvement is observed in 52\% to $76 \%$ of Issiaka M | Volume 2; Issue 3 (2021) | JOAR-2(3)-027 | Case Report

Citation: Issiaka M, et al. Peripheral Ulcerative Keratitis: Another Ocular Manifestation of Behçet's Disease. J Ophthalmol Adv Res. 2021;2(3):1-5. 
patients [5]. The characteristic feature is recurrent uveitis that may involve the anterior and/or posterior segment. Corneal symptoms are becoming more common and have been the topic of current research, such as Peripheral Ulcerative Keratitis (PUK), bilateral small ulcerative keratitis $[1-3,6]$. It is, in fact, a crescent-shaped stromal inflammation affecting the adjacent corneas, with sectoral thinning of the afflicted region. Our patient has a similar case with a pseudo-Mooren-like marginal ulcer and this interval of healthy peri-limbal corneal zone.

The etiopathogeny of this condition seems to have been described by several authors. Several attacks at various levels have been reported. Gunduz, et al., reported that tear film function is decreased in patients with Behçet's disease compared to healthy controls [7]. This is a qualitative alteration of the tear film. Also, Smith VA, et al., describe that Matrix Metalloproteinase (MMP) activity is upregulated in Behcet's disease and that mainly MMP-2 and MMP-9 seem to be involved in the pathology of the early phase of peripheral ulcerative keratitis [8]. Thus, it is a mechanism maintained by two phenomena: keratitis can develop or worsen either by the decrease in tear film quality or by the upregulated activity of MMPs, or both. We also consider the possibility of these two phenomena together in our patient, in whom we find the presence of moderate dry eye with a severely altered tear film that responded well to replacement therapy with wetting agents, although we did not use the study of matrix metalloproteinase activity. Pharmacological agents utilized for the treatment of BS include corticosteroids, colchicine, azathioprine and Tumour Necrosis Factor (TNF) $\alpha$ inhibitors, among others [4]. Management is based on the treatment of the causative disease and local treatment (corticosteroid therapy $+/$-topical cyclosporine). Local corticosteroid therapy is the first-line treatment for immunologic ulcers. But in the case of insufficient response, topical or oral cyclosporine is used. Yong-Sok Ji reported a case that progressed well with a combination of corticosteroid therapy (local prednisolone acetate $1 \%$ and oral); cyclosporine A $1 \%$ (topical at 6 instillations per day and oral) and sodium hyaluronate $0.3 \%$ [2]. In our patient, management with corticosteroid therapy (local and oral) plus wetting agents was sufficient with dramatic improvement. In addition to traditional steroid treatment, an immunosuppressant such as antitumor necrosis factor-alpha (Anti-TNF alpha) has been included in the treatment of ocular inflammatory disease [9]. The prognosis is favorable if the management is prompt and adequate. It is important to remember to involve the internist in the overall management of Behçet's disease.

\section{Conclusion}

This case illustrates once again the possibility of corneal involvement revealing ocular involvement in Behçet's disease and suggests then screening and treat any dry eye, even minimal, in these patients. The peripheral location of corneal ulcers should raise the possibility of autoimmune etiology and suggest corticosteroid treatment or, in case of resistance, local or systemic immunosuppressive drugs. 


\section{Conflict of Interest}

The authors declare no conflict of interest, financial or otherwise.

\section{References}

1. Jinagal J, Agarwal A, Negi A, Gupta G, Sharma V, Gupta PC, et al. Immune keratitis: An unusual primary presentation of neuro-Behçet's disease. Eur J Ophthalmol. 2019;29(4):NP5-8.

2. Ji YS, Yoon KC. A rare case of peripheral ulcerative keratitis associated with Behçet's disease. Int Ophthalmol. 2014;34(4):979-81.

3. Zeng, J, Chen, B. Severe primary ocular surface involvement in Behcet disease. Optom Vis Sci. 2014;91:e301-4.

4. Dalvi, SR, Yildirim, R, Yazici, Y. Behçet's syndrome. Drugs. 2012;72:2223-41.

5. Kontogiannis V, Powell RJ. Behçet's disease. Postgrad Med J. 2000;76(900):629-37.

6. Murphy C, Gregory ME, Ramaesh K. Peripheral ulcerative keratitis: an unusual primary ocular manifestation in Behcet's disease? Rheumatology (Oxford). 2009;48(12):1490.

7. Gunduz A, Cumurcu T, Demirel EE, Akpolat N, Karincaoglu Y. The ocular surface in the Behcet's disease patient. Can J Ophthalmol. 2012;47:429-34.

8. Smith VA, Hoh HB, Easty DL. Role of ocular matrix metalloproteinases in peripheral ulcerative keratitis. $\mathrm{Br}$ J Ophthalmol. 1999;83(12):1376-83.

9. Neri P, Zucchi M, Allegri P, Lettieri M, Mariotti C, Giovannini A. Adalimumab (Humira ${ }^{\mathrm{TM}}$ ): a promising monoclonal anti-tumor necrosis factor alpha in ophthalmology. Int Ophthalmol. 2011;31(2):165-73. 